\title{
PALS Syndrome Post-LASIK
}

\author{
Noel Alpins ${ }^{1,2}$ and George Stamatelatos ${ }^{2}$
}

1. Department of Ophthalmology, Melbourne University, Melbourne, Australia; 2. NewVision Clinics, Melbourne, Australia

\begin{abstract}
A 34-year-old male presented complaining of ongoing symptoms of glare, ghosting, starbursts, haloes, and reduced contrast sensitivity commonly referred to by the acronym "GASH". His unaided visual acuity achieved was a satisfactory 20/20 in each eye. To reduce his symptoms and enable driving at night, he must resort to shining the torch from his cell phone into his eyes to constrict his pupils. These symptoms are due to excess corneal astigmatism remaining, and is prevalent post refractive laser surgery when treatment is based on refractive parameters alone without any regard for corneal measures and having ocular residual astigmatism greater than 1.00D preoperatively. In this commonly prevalent clinical situation, corneal astigmatism postoperatively will be more than 1.00D, together with a significant risk of one or more symptoms of glare, starbursts, or haloes which, when they occur together, is known as predictable avoidable laser-assisted in situ keratomileusis surprise (PALS) syndrome.
\end{abstract}

DOI: https://doi.org/10.17925/USOR.2019.12.1.37

\section{Keywords}

ORA, ocular residual astigmatism, vector Planning, glare, starbursts, haloes

Disclosures: Noel Alpins and George Stamatelatos report a financial interest in ASSORT Surgical Management Systems which holds trademarks in Vector Planning ${ }^{\text {TM }}$

Review Process: Double-blind peer review.

Compliance with Ethics: All procedures were followed in accordance with the responsible committee on human experimentation and with the Helsinki Declaration of 1975 and subsequent revisions, and informed consent was received from the patient involved in this case study.

Authorship: All named authors meet the International Committee of Medical Journal Editors (ICMJE) criteria for authorship of this manuscript, take responsibility for the integrity of the work as a whole, and have given final approval to the version to be published.

Received: March 7, 2019

Accepted: March 27, 2019

Citation: US Ophthalmic Review. 2019;12(1):37-9

Corresponding Author: Noel Alpins, NewVision Clinics, 160 Victoria Parade, East Melbourne, Victoria 3002, Australia. E: alpins@newvisionclinics.com.au

Support: No funding was received in

the publication of this article.
A 34-year-old male presented to the clinic for a second opinion regarding ongoing symptoms of glare, ghosting, starbursts, haloes and reduced contrast sensitivity three years post-laser-assisted in situ keratomileusis (LASIK) surgery. These symptoms were exacerbated under low light conditions, particularly driving at night, and he found a reduction in their effect when shining his cell phone torch light in his eyes.

Preoperatively, manifest refraction was: right -3.25/-0.75 x 33, left -3.75/-0.50 x 152. His cycloplegic refraction was: right $-2.50 /-1.00 \times 20$ and left $-3.00 /-0.75 \times 165$.

Simulated keratometry from topography preoperatively was: right 41.00/42.50 @ 102 and left 40.75/42.25 @ 75 with regular corneas in both eyes (Figure 1).

Pupil diameter under mesopic conditions was measured as: right $6.37 \mathrm{~mm}$ and left $6.94 \mathrm{~mm}$.

Uncomplicated bilateral LASIK surgery was performed by another clinic in July 2016 using the latest generation Schwind Amaris ${ }^{\circledast}$ 1050RS Hz excimer laser (Schwind eye-tech-solutions GmbH \& Co. KG, Kleinostheim, Germany) with the treatment based on the manufacturer recommended practice of manifest refraction only. The optical and the ablation zones were $6.70 \mathrm{~mm}$ and $7.63 \mathrm{~mm}$, respectively.

At the 7-month postoperative review, unaided visual acuity was 20/20 part in both eyes and 20/15 with a manifest refraction of right plano/-0.75 $\times 9$ and left plano/-0.75 × 170. Bilateral retreatment by flap lift was then performed based again on the manifest refraction, only because of the symptoms and remaining minor refractive error.

The patient attended our clinic 2 years post initial LASIK, complaining of significant symptoms of glare, ghosting, starbursts, haloes, and reduced contrast sensitivity (GASH), and wanted a second opinion on reducing these effects, which presented after the initial refractive laser surgery. On examination, the unaided visual acuity of right 20/20 and left 20/20-2 best correcting to 20/20 in both eyes. Manifest refraction right plano and left plano/-0.50 × 180. Cycloplegic refraction of right $+0.25 \mathrm{DS}$ and left $+0.50 /-0.25 \times 180$. Corneal astigmatism using simulated keratometry from the CSO Sirius tomographer (C.S.O. Srl, Firenze, Italy), was right 1.12D @ 90 and left 1.04D @ 85. Ocular wavefront using the ViSX Wavescan system showed higher order aberrations of right 0.27 microns and left 0.40 microns.

It is interesting to note that there was still a significant amount (>1.00D) of corneal astigmatism remaining in each eye after both the first treatment and the second enhancements, which were based on the manifest refraction parameters alone. Treating the maximum amount of astigmatism 
Figure 1: Preoperative axial curvature topography displaying regular with-the-rule astigmatism for right and left eyes

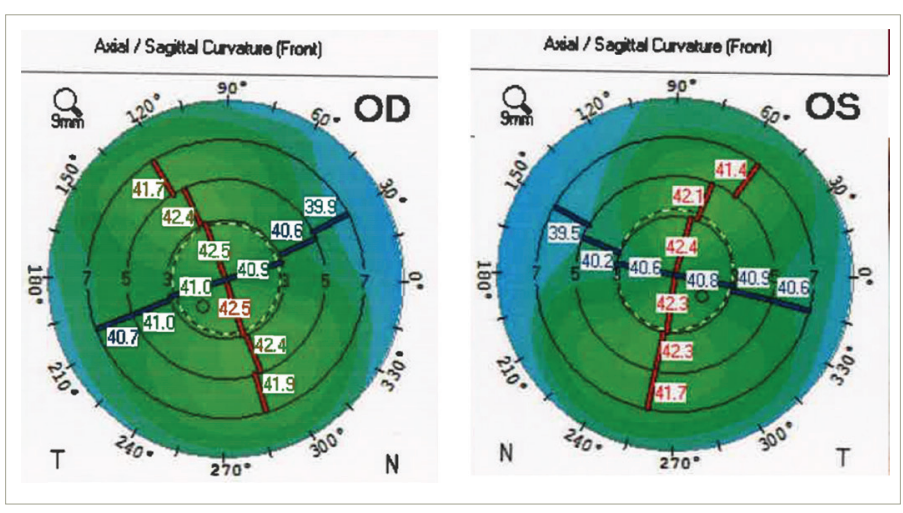

by incorporating the corneal parameters into the refractive treatment plan employing Vector Planning ${ }^{1}$ would have left less corneal astigmatism postoperatively and reduced symptoms of glare, ghosting, starbursts, and haloes.

\section{Ocular residual astigmatism}

To treat the maximum amount of astigmatism, calculation of the ocular residual astigmatism (ORA) is required in each case. The ORA is defined as the vectorial difference between the corneal astigmatism and the refractive cylinder at the corneal plane. ${ }^{1}$ It is expressed in dioptres together with an axis and has been shown to be more than 1.00D in $46 \%$ of cases $^{2}$ in one study and $34 \%$ of cases in another study. ${ }^{1}$

In this case, the ORA preoperatively was right 0.99D Ax 6 and left 1.24D Ax 174 (Figure 2). Due to the pre-existing corneo-refractive differences the ORA is the minimum amount of astigmatism that can remain postoperatively. Treatment based on the refractive parameters alone, as was the case here, would leave all the ORA, theoretically 0.99D @ 96 for the right eye and $1.24 \mathrm{D} @ 84$ for the left eye to be directed to the cornea postoperatively. Note that this is 90 degrees away from the ORA axis to neutralise it. Even after the second treatment, which again was based on refractive parameters to reduce the refractive cylinder, there was still predictably a substantial amount of corneal astigmatism (>1.00D) remaining: right 1.12D @ 90 and left 1.04D @ 85 due to the significant amount of ORA.

Incorporating the corneal parameters into the refractive treatment plan after calculating the ORA would treat the maximum amount of astigmatism and leave less corneal astigmatism postoperatively compared to treatment based on refractive parameters alone with the same amount of refractive cylinder remaining. ${ }^{3}$ Vector Planning is a systematic method of combining both refractive and corneal parameters into the excimer treatment plan. This has been shown to reduce the corneal astigmatism remaining compared to treatments based on refractive parameters alone without compromising the refractive cylinder postoperatively. ${ }^{3}$

\section{Vector Planning}

The surgeon decides how to apportion the preoperative ORA calculated by placing an emphasis of corneal to refractive parameters anywhere from $1 \%$ corneal astigmatism and $99 \%$ refractive cylinder to $99 \%$ corneal and
Figure 2: The ocular residual astigmatism is calculated as the vectorial difference between manifest refractive cylinder at the corneal plane and corneal astigmatism

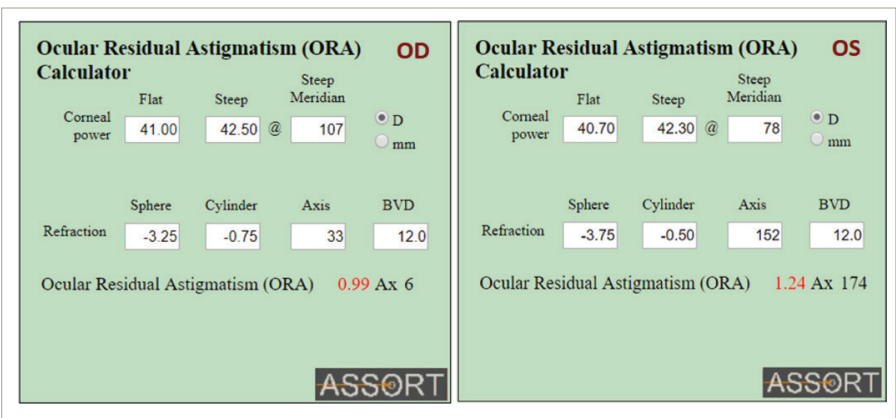

Figure 3: Calculation of ocular residual astigmatism for the right eye

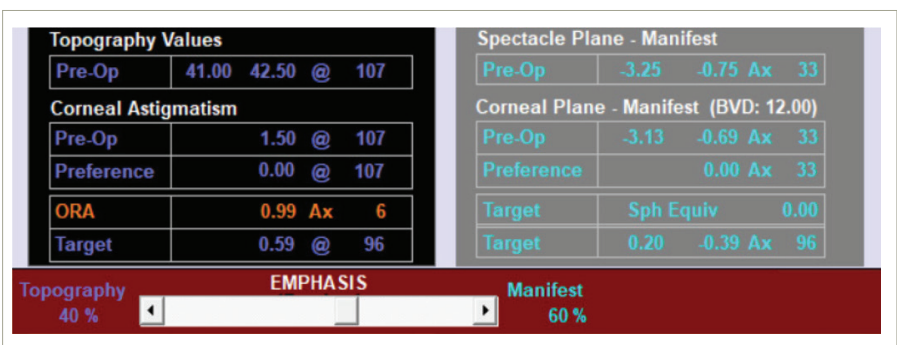

Vector Planning places emphasis of $40 \%$ topography and $60 \%$ manifest refraction on the ocular residual astigmatism to target 0.59D of corneal astigmatism.

Figure 4: Calculation of ocular residual astigmatism for the left eye. Vector Planning places emphasis of $40 \%$ topography and $60 \%$ manifest refraction on the ocular residual astigmatism to target $0.74 \mathrm{D}$ of corneal astigmatism

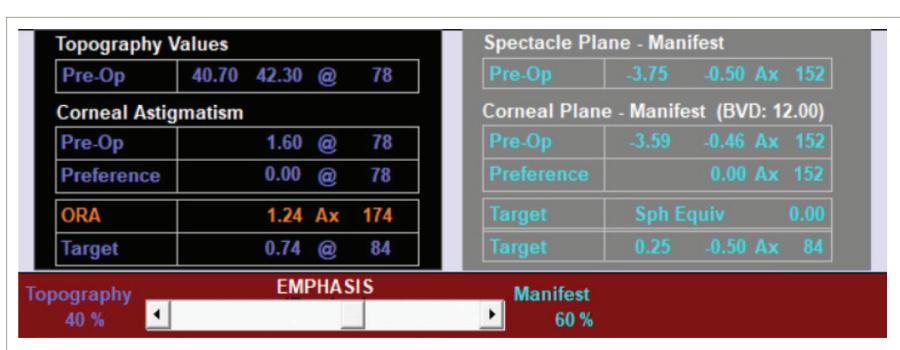

$1 \%$ refractive emphasis. Studies using the method of Vector Planning ${ }^{3-5}$ have shown $40 \%$ emphasis on corneal parameters and 60\% emphasis on refractive parameters to be suitable for most treatments with a range anywhere from $45-65 \%$ emphasis by refractive parameters. The important consideration is the ORA, knowing that any emphasis on the ORA is treating the maximum amount of astigmatism.

Applying Vector Planning to this case study, the ORA calculated for the right eye is 0.99D Ax 6. By emphasising, for example, $60 \%$ of the ORA towards refraction and 40\% towards topography, 0.59D @ 96 would be targeted on the cornea and $-0.39 \mathrm{D} \times 96$ in the refraction (Figure 3). This compares to $1.12 \mathrm{D} @ 90$ of corneal astigmatism that the patient has postoperatively. 
For the left eye the ORA is $1.24 \mathrm{D} @ 84$. So again, emphasising the ORA by $60 \%$ towards refractive parameters and $40 \%$ by topography, the targeted corneal astigmatism would be $0.74 \mathrm{D} @ 84$ and the refractive cylinder $-0.50 \times 84$ (Figure 4). This compares to 1.04D @ 85 of corneal astigmatism that the patient now has postoperatively.

Vector Planning targets a spherical equivalent of zero in the refraction and because of the better corneal shape that the patient is left with postoperatively, compared to treatments based on refractive parameters alone, the targeted refractive cylinder has been shown not to be accepted by the patient in the postoperative manifest refraction.

Unfortunately, the patient did not want to undergo any further (third) surgery to reduce his symptoms, but was advised that Vector Planning was a viable option.

Excess corneal astigmatism is prevalent post refractive laser surgery with many complaints now documented in letters to the US Food and Drug Administration and the New York Times of symptoms of glare, ghosting, starbursts, haloes, or decreased contrast sensitivity, now commonly termed $\mathrm{GASH}^{6}$ as the acronym to describe these symptoms.
The method of Vector Planning can address the excess corneal astigmatism causing these disturbing symptoms and improving patient vision quality and satisfaction rates post-LASIK. The prevalence of these symptoms and signs together qualifies it as a syndrome. An ORA of more than $1.00 \mathrm{D}$ preoperatively and corneal astigmatism of greater than 1.00D postoperatively, together with any one or more of the GASH symptoms constitutes preventable avoidable LASIK surprise (PALS) syndrome. This syndrome is preventable by calculating the ORA preoperatively to ascertain how much corneo-refractive difference there exists. It is avoidable using the method of Vector Planning to reduce excess corneal astigmatism postoperatively, it can validly apply to photorefractive keratectomy and small incision lenticule extraction as well as LASIK procedures and the surprise of GASH can be minimized or eliminated by maintaining postoperative corneal astigmatism at less than 1.00D.

Laser manufacturers need to incorporate the Vector Planning method as a standard function of their laser systems, allowing all surgeons this option to maximally treat astigmatism and prevent PALS syndrome. This is currently not available on any laser. For surgeons who want to avail themselves of this facility of ORA calculation and Vector Planning with their refractive laser surgery, this is available as a free application at www.assort.com. $\square$
1. Alpins NA. New method of targeting vectors to treat astigmatism. J Cataract Refract Surg. 1997;23:65-75.

2. Frings A, Katz T, Steinberg J, et al. Ocular residual astigmatism: Effect of demographic and ocular parameters in myopic laser in situ keratomileusis. J Cataract Refract Surg. 2014:40:232-8.

3. Arbelaez MC, Alpins N, verma S et al. Clinical outcomes of laser in-situ keratomileusis with an aberration -neutral profile centred on the corneal vertex comparing vector planning with manifest refraction planning for the treatment of myopic astigmatism. J Cataract Refract Surg. 2017;43:1504-14.

4. Alpins NA, Stamatelatos G. Customized PARK treatment of myopia and astigmatism in forme fruste and mild keratoconus using combined topographic and refractive data. I Cataract Refract surg. 2007;33:591-602.
5. Alpins NA, Stamatelatos G. Clinical outcomes of laser in situ keratomileusis using combined topography and refractive wavefront treatments for myopic astigmatism. J Cataract Refract Surg. 2008:34:1250-9.

6. LASIK Complaints Filed With the FDA. Available at: www.lasikcomplications.com/lasik-complaints.htm (accessed February 20, 2019). 